\title{
Prefilled syringe: a review of injectable dosage form delivery system
}

\author{
Sandip Arun Borse, Ashish Prakash Gorle*, Kuldip Patil. \\ R. C. Patel Institute of Pharmaceutical Education and Research, Shirpur. (MS) \\ (sandipaborse29@gmail.com,gorleashish25@gmail.com,kuldiprajput1011@gmail.com )
}

\begin{abstract}
:
Prefilled syringe are convenient device for the drug delivery of parental dosage form. There is easy to makes and delivering the precise dosage of the medication. The content of this review article is component of Prefilled syringe, application of Prefilled syringe, compatibility study of the drug product with the packing material, Extractable and leachable and challenge's with the Prefilled syringe. This type of knowledge can familiarize the formulation scientist. In the use of Prefilled syringe as both packing and delivery system for pharmaceutical drug product is accelerating. Prefilled syringe must meet the quality, stability and efficacy for both system including with the drug product. In that, the incompatibility study between the drug product and Prefilled syringe including the safety of the drug product. In that the ability of the extractable and leachable to interact with the drug product ingredients and they can affect the safety, efficacy and stability of the drug product.
\end{abstract}

Keywords: Extractable, leachable, prefilled syringe, incompatibility, autoinjector, silicone oil.

\section{Introduction}

The improvement of pre-filled syringes (PFS) in the mid-twentieth century has changed the point of view of human services experts. Parenterals are essential decision for doctors needing quick acting, safe medication conveyance. The parenteral medication part has developed as of late with the improvement of new biopharmaceuticals for a scope of intense and interminable conditions including diabetes, development lacks, numerous sclerosis, rheumatoid joint inflammation, haemophilia, malignancy and numerous different illnesses. In-accordance with this development, there has been an impressive improvement in pre-filled syringes and other pre-filled conveyance gadgets. Pre-filled syringes are infusion gadgets that expel the filling step from another compartment, for example, a vial or ampoule. Fusing the pre-filled syringe into a propelled conveyance gadget, for example, a pen or autoinjectors can bring extra client advantages, for example, auto needle addition, programmed conveyance of the medication and safe removal of the needle after use. These highlights open up the open door for more extensive utilization of self-organization of medications at home, which can improve consistence and decrease the time and cost of organization by medicinal services expert.

Right now, assess this adjustment in situation from traditional dose structures to PFSs corresponding to improvement of details, similarity issues, human services and patient security perform. A move towards dispensable gadgets has improved the comfort of self-overseeing the right measure of medication a few times each day. Current gadgets have advanced to give an elevated level of client acknowledgment. The development rate in pen gadgets is considerably more noteworthy than for syringes. The utilization of reusable and expendable autoinjectors to regulate medications to treat a scope of constant sicknesses, for example, different sclerosis and rheumatoid joint pain has additionally expanded in the course of recent years. Various investigations have been distributed that show improvement in persistent inclination and execution equivalent with that of an infusion regulated by a social insurance proficient. All the more as of late in exceptionally serious markets, for example, rheumatoid joint pain, new items have been at first propelled in autoinjector gadgets - a pattern that is set to develop as cutting edge infusion gadgets become all the more generally utilized.

The popularity of pre-filled syringes and advanced injection devices is increasing rapidly, biopharmaceuticals and vaccines $[1,2]$. The main reasons for this increase in popularity of the pre-filled syringe format include the following:

- Reduced risk of contamination

- Reduced handling requirements

- $\quad$ Ease and speed of administration

- Potential for self-administration by the patient

- Improved patient compliance

- Sterility assurance

- Added convenience through combination with an autoinjectors (Figure 2A)

- Elimination of the need for overfill of costly therapeutics and consequent reduced waste 
- Minimisation of dosage errors due to the reduced number of steps involved

The utilization of pre-filled syringes for parenteral organization of drugs has developed significantly. Their utilization will probably keep on becoming because of their numerous focal points for the patient just as for assembling. Focal points for the patient incorporate simplicity of self-organization and a diminished danger of defilement because of the less advances required for arrangement of the portion when contrasted and prescriptions filled into vials. The preferences during assembling incorporate protection of medication arrangement since next to zero overload volume is required in the essential compartment and less planning is required for assembling when the syringes are gotten pre-sanitized.

The as of late expanded enthusiasm for prefilled syringes (PFS) is to a great extent driven by numerous favourable circumstances against conventional ampoules and vials, for example, permitting brisk and precise dosing, limiting dosing blunders, decreasing the danger of natural pollution, upgraded comfort and usability, forestalling of overload, etc. On expanding number of accessible natural medications, the interest for the utilization of PFS has extensively expanded as of late.

Numerous biotech tranquilize items are lyophilised in vials because of their poor strength for parenteral organization. Be that as it may, the advancement of fluid definitions of biotech items applying prefilled syringes has been expanding quickly, determined additionally by improved security being used, client comfort and simplicity of organization. Another significant perspective is the move from emergency clinic treatment to homecare and patient self-infusion for some ceaseless infections and explicit remedial territories.

Today, $85 \%$ of human services proficient incline toward PFSs over other parenteral conveyance frameworks [3]. Glass syringes pre-loaded up with drugs or organic items are mix items under 21 CFR Part 3. In particular, they meet the mix item definition under 21 CFR[4].

\section{COMPNENTS OF PREFILLED SYRINGE}

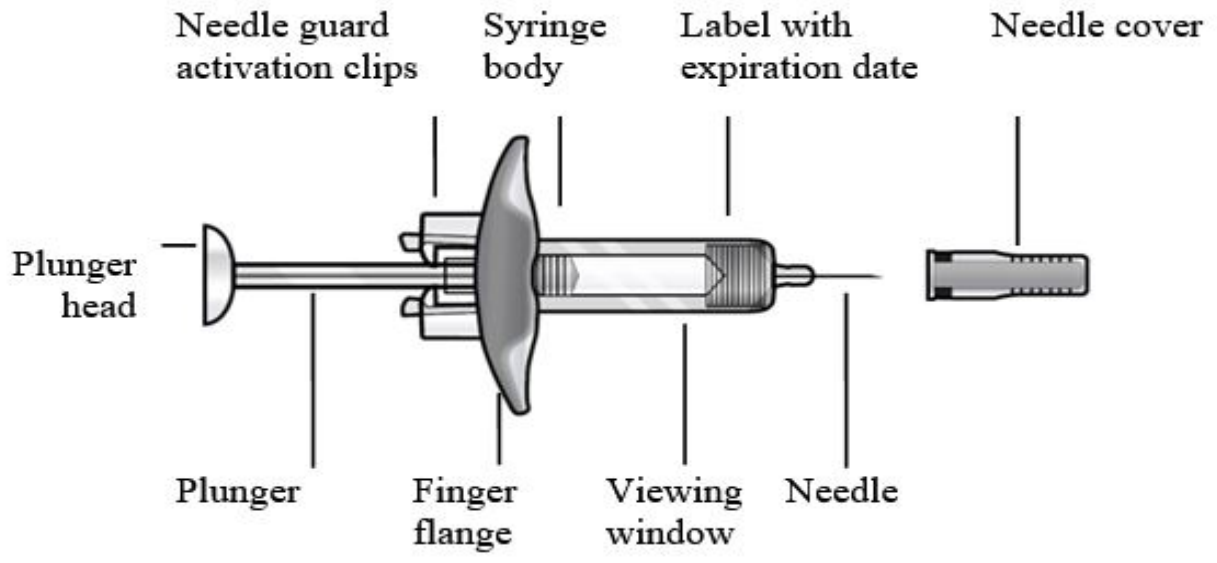

\section{TYPES OF PREFILLED SYRINGE}

\section{GLASS based system}

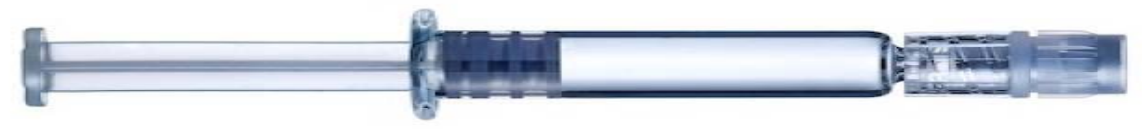




\section{PLASTIC based system}

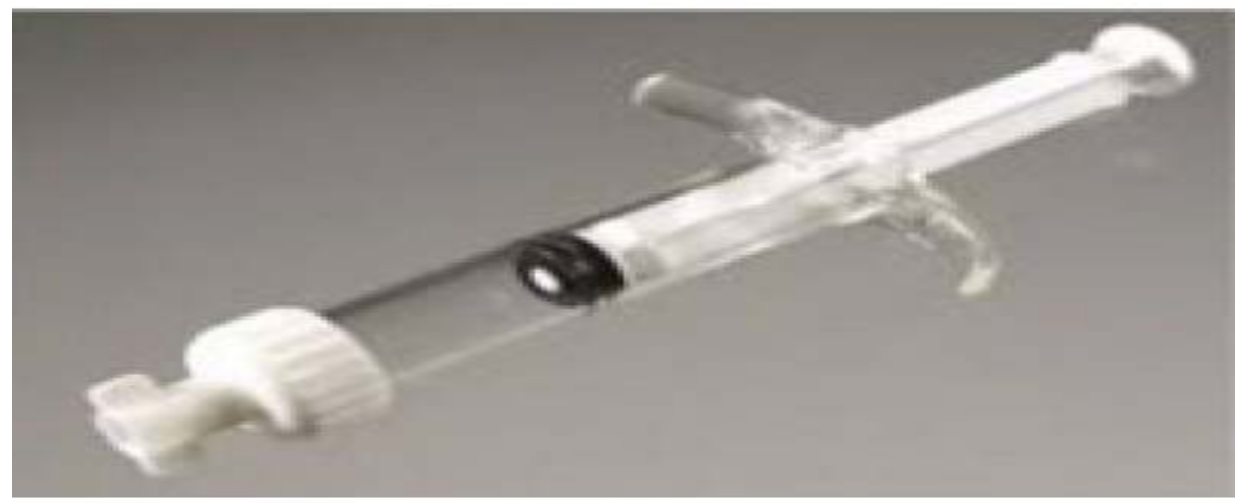

The decision of glass or plastic for the manufacture of the prefilled syringes is turning into an inexorably significant issue. Most pre-filled syringes in the European and US markets are made of glass. Until quite a while prior, plastic materials utilized for syringe barrels, for example, polypropylene, polyethylene and polycarbonate had the disservice of not being as straightforward as glass making the visual examination of substance troublesome. Be that as it may, 'glass-like' cyclic olefin copolymer (COC) and cyclic olefin polymer (COP) have as of late been created. These materials have astounding straightforwardness [5] great dampness boundary properties, low surface authoritative and low degrees of extractables [6]. They, along these lines, present solid rivalry to customary glass syringes. All things considered, the COC and COP materials will continuously build the portion of polymer materials in the pre-filled syringe showcase. While quite a long while prior polymer prefilled syringes were impressively more costly than their glass reciprocals, the distinction in cost has been diminished fundamentally. The decision among glass and plastic depends on their material attributes (Table 1) but on the other hand is influenced by the commonness of assembling lines used to fill and finish glass syringes. This clarifies the generally little extent of plastic syringes right now present in many markets. The determination of the elastomeric material for the unclogger is likewise significant, and various physical properties must be thought of, including versatility, hardness, penetrability to oxygen and water fume just as explicit extractables and leachables. Some of this information are given by the producers [7].

\section{ADVANTAGES OF PREFILLED SYRINGE:}

- $\quad$ Pre-filled syringes make injections easier and safer for both doctors and patients.

- In the administration of pre-filled syringe, a patient always receives the right dosage.

- Pharmaceutical companies can benefit from less overfill in pre-filled syringes.

- Pre-filled syringes work well with increasingly popular safety devices and auto-injection systems.

\section{DISADVANTEGES OF PREFILLED SYRINGE}

- The preparation of prefilled syringe ensure patient safety by demonstrating that no component or material has any extractables and leachables.

- The primary packaging does not have an adverse interaction with the drugs.

- Some of the extractables and leachables can interfere with the drug molecules and could compromise the effectiveness of a drug.

- In the prefilled syringe drug is contact with all component and chances to extractables and leachables problem.

\section{Application of Prefilled Syringes}

Prefilled syringe frameworks need to meet different necessities and functionalities, for example holder conclusion uprightness, heat opposition, stun obstruction, unclogger coasting powers, squander removal, etc. Prefilled syringes comprise of different parts and materials, for example, glass, polymers and elastomers, which must be chosen properly to guarantee they meet the prerequisites for their proposed use (Figure 1).

In creating prefilled syringe frameworks, different advancements are thought of, for example, item configuration, contact surface treatment and materials to fulfill quality necessities for infusion. A few productions have detailed explicit quality issues with biomolecules in prefilled syringe frameworks. Conglomeration of restorative proteins is one of the most basic hazard factors since it might affect adversely on viability and security because of the protein deactivation and insusceptible reactions in patients. 
A significant issue is silicone oil-incited total. Silicone oil has been utilized as oil to accomplish smooth unclogger coasting usefulness. In any case, with regards to sedate atom, silicone oil turned into a difficult issue since it can actuate aggregation. Furthermore, the prefilled syringe fabricating process is viewed as a potential hazard factor. For example, tungsten pins are utilized for the glass barrel tip forming process. Protein conglomeration within the sight of tungsten has been observed.

\section{Compatibility of the drug product with the packaging system}

Parenteral definition comprises of the segments of the medication item just as the bundling framework [8]. Association between the medication item and different segments of the bundling framework can be basic for item soundness. Along these lines, guaranteeing the similarity of the medication item with the last bundling framework is a fundamental piece of the item improvement. The assessment of the collaborations with the compartment conclusion framework, including adsorption and corruption of the dynamic pharmaceutical item just as debasement of the holder during capacity, is laid out in FDA rules [8]. A basic piece of the similarity evaluation is the comprehension of the nature and effect of extractables and leachables from the bundling material on the medication item. Extractables are mixes discharged from a bundling part under forceful treatment conditions surpassing those the segment may suffer during ordinary

use. Leachables are a subset of extractables that are freed from the bundling or naming segments into the medication item under ordinary conditions [8]. Leachables can start from any material that comes into contact with the medication item. As plot in the applicable administrative rules, any contact between the bundling framework and the medication item gives a chance to the item to be changed [9]. Because of the higher unpredictability of the bundling framework, the quantity of intensifies that may filter into the medication item arrangement is extensively higher because of pre-filled gadgets contrasted and vial-based introductions [10]. An all-around detailed case of an incongruence issue that was most likely due to leachables and prompted antagonistic clinical impacts and item disappointment in a pre-filled syringe design.

Notwithstanding evaluating the effect of leachable segments on tolerant wellbeing, the possibility to initiate or quicken protein corruption should be considered during plan improvement. Given the expanding decision of bundling materials, there is a solid need to create exhaustive, institutionalized procedures for evaluation of extractables and leachables. A genuine case of such an appraisal program, in light of choice of bundling segments, investigation of extractables, and wellbeing based evaluation of leachables. A portion of the key connections between the medication item and the holder conclusion frameworks are talked about underneath.

\section{Interaction with Silicone Oil}

Silicone oil is applied to cartridges and barrels of syringes to encourage smooth and simple development of uncloggers inside the syringe barrel. Under average conditions, protein arrangements in pre-filled syringes can be presented to a few sorts of pressure factors, for instance, shaking during transportation and temperature journeys while in contact with the silicone oil. Along these lines, it is fundamental to evaluate similarity of a protein definition with this oil during the improvement of the detailing for use in pre-filled syringes. The measure of silicone oil applied to a pre-filled syringe is commonly kept to the least sum conceivable and goes between about 0.6 and $1.0 \mathrm{mg}$ per $1 \mathrm{ml}$ syringe [8]. Since pre-filled syringes act both as a conveyance gadget and as a capacity compartment, proteins planned in pre-filled syringes are presented to silicone oil- - water interfaces for their whole time span of usability. Silicone beads can likewise be discharged into the medication item. There are two kinds of strategies for use of silicone oil to syringes, showered on and heated on. The heated on application contains impressively less free silicone oil and for the most part brings about lower discharge into the medication item [11]. Subsequently, it speaks to a lower hazard from the advancement point of view when choices about the holder conclusion framework are made [12]. Silicone oil beads, which relocate into arrangement, are commonly somewhere in the range of 1 and $25 \mu \mathrm{m}$ however can likewise be littler than $1 \mu \mathrm{m}$ [13]. More silicone is in truth discharged into the medication item during the infusion than during the capacity, despite the fact that syringe tumult during capacity diminishes the distinction between these two qualities. It was likewise assessed that the absolute zone of silicone oil on the barrel is three sets of size higher than the surface territory of beads in arrangement [13]. There are two potential issues related with silicone oil. In the first place, communications between silicone oil and proteins can diminish dependability of the protein therapeutics and second, the arrival of silicone beads expands the sub-noticeable molecule include in the detailing. Cases have been accounted for where silicone oil used to cover pre-filled syringes filtered into insulin arrangements, bringing about arrangement darkness and obvious insoluble protein totals with resulting loss of insulin movement [14]. The shadiness was credited to insulin particulate development brought about by the sullying of the arrangement by silicone oil. Collection of an enemy of streptavidin IgG1 monoclonal immune response was likewise seen within the sight of generally high $(1.5 \% \mathrm{w} / \mathrm{v})$ levels of silicone oil; notwithstanding, disturbance was required as an extra pressure factor [15]. So also elevated levels of silicone oil $(5 \mathrm{mg} / \mathrm{ml})$ were appeared to cause total of four proteins, ribonuclease A, lysozyme, ox-like serum egg whites and concanavalin A, without surfactant. No impact of 
silicone oil on optional and tertiary structure was seen right now. It ought to be noticed that both of these investigations depended on spiking explores different avenues regarding silicone oil focuses a lot higher than restorative proteins may commonly be presented to in a pre-filled syringe. A blend of the nearness of silicone oil and fomentation stress was accounted for to be important to cause conglomeration.

The analyses were attempted in business pre-filled syringes to think about the impact of the syringe surfaces (silicone oil, glass and BD-42 covering). The impact was seen as $\mathrm{pH}$ subordinate, demonstrating the significance of plan advancement for control of the collaborations with silicone oil. The nearness of a surfactant can affect the protein security within the sight of silicone oil. Without polysorbate 80 , a quantifiable loss of albinterferon alfa$2 \mathrm{~b}$ from the arrangement stage was accounted for within the sight of an elevated level of silicone oil [16]

As the silicone oil fixation expanded, the measure of protein in arrangement after centrifugation diminished, recommending upgraded protein relationship with silicone oil. Within the sight of polysorbate 80 , no misfortune in protein in the arrangement stage happened, demonstrating immaterial authoritative of albinterferon alfa- $2 b$ to silicone oil. This is steady with late work utilizing BSA, lysozyme, trastuzumab and abatacept proteins showing that the expansion of $0.03 \%(\mathrm{w} / \mathrm{v})$ polysorbate 20 diminished adsorption of proteins to silicone oil micro droplets in a $1 \%$ silicone emulsion arrangement [17]. This was in all likelihood because of particular adsorption of polysorbate 20 to the silicone oil- - water interface found that a monoclonal counter acting agent sedate item displayed more noteworthy degrees of particles more prominent than $10 \mu \mathrm{m}$ and turbidity after capacity in prefilled glass syringes contrasted and capacity in glass vials. All other dependability traits including dissolvable totals, immaculateness and charge variations stayed unaltered. Comparative outcomes, utilizing showered on prefilled syringes. Be that as it may, no expansion in the molecule tally was watched utilizing prepared on silicone pre-filled syringes. The degrees of particulate issue in pre-filled syringes still ordinarily fall impressively beneath as far as possible as characterized by the US Pharmacopeia [16]. In general, it creates the impression that as a rule silicone oil does not present genuine down to earth, wellbeing or adequacy issues if plan is streamlined for the particular application. From a useful point of view, the refered to reports demonstrate that streamlining of essential plan parameters, for example, $\mathrm{pH}$ and ionic quality, together with the nearness of a surfactant, are the best methodologies for controlling the connections between protein therapeutics and silicone oil. Given its long history of security, biocompatibility and dependability, silicone oil is an adequate oil for pre-filled gadgets. Be that as it may, if limits are set later on for particles littler than $10 \mu \mathrm{m}$, the nearness of silicone beads may should be additionally tended to. Silicone oil can be subbed by elective coatings, for example, fluorocarbon coatings. Be that as it may, subbing silicone oil with different substances, for example, PTFE was appeared to cause protein collection because of adsorption of protein atoms at the strong - fluid interface [18].

\section{Interaction with Tungsten species}

Tungsten pins are routinely utilized in the glass syringe fabricating procedure to keep the drag open while the cone is being molded. During this procedure, particles of tungsten and tungsten oxide can be kept inside the syringe. At the point when presented to an acidic $(\mathrm{pH}<6)$ watery arrangement, different solvent polyanionic species can be framed out of both basic tungsten and tungsten oxides. These can possibly initiate protein oxidation, collection and arrangement of proteinaceous particles [19,20]. This incompletely reversible communication is essentially electrostatic in nature and reliant on the centralization of both protein and tungsten [21]. The impact is frequently tried by spiking plans with dissolvable tungsten species. A spiking study exhibited an expanded inclination of Epoetin alpha to total within the sight of sodium polytungstate (2-20 ppm). The impact was fixation subordinate, being the most noteworthy at the most noteworthy polytungsten focus. In any case, the degree of tungsten was well over that normal in the pre-filled syringes. Different types of tungsten, for example, tungsten (IV) oxide or tungsten (VI) oxide did not show a comparable impact. Protein precipitation within the sight of polytungstate as a charge-nonpartisan complex was additionally observed with the fermented Na2WO4 tungsten species being the most powerful [22]. In another examination, it was exhibited that spiking a protein arrangement with a concentrate from tungsten pins brought about more prominent degree of protein debasement than utilizing dissolvable tungsten species, for example, tungsten (VI) oxide [19]. The debasement impact of tungsten particles on protein therapeutics can be balanced through modification of detailing $\mathrm{pH}$ just as protein focus and ionic quality. By and large, be that as it may, the quantity of detailed tungsten incongruencies in organic items at tungsten focuses important for pre-filled syringes is low[30].

\section{Extractables and leachables}

Extractables are substance build-up, both natural and inorganic, that can separate out in the detailing from the segment of compartment conclusion framework or gadget. Leachables are substance deposits that move to the definition over self-existence of the item. Extractables for the most part happen because of connection of item with bundling material under outrageous states of temperature or solvents. Leachables can be said to be the subset of extractables. Distinction in these two can be said as in various removing condition and time allotment [23]. 
Extractables and leachables are developing worries for pharmaceutical makers and administrative specialists. A comparable explanation is written in Code of Federal Regulation (21 CFR 211.94) that states insights about medication item compartments and terminations. Likewise, both the United States Pharmacopeia (USP) and European Pharmacopeia contain sections that manage the testing of plastic materials, including extraction tests. Notwithstanding, restricted rules are accessible for Extractables and leachables considers. The most important rule for Extractables and leachables examines is the European Medicine Agency rule that was affirmed in May 2005 in London by the Committee for Medicinal Products for Human use. It orders extraction reads for the utilization of non-compendial plastic material utilized for compartment conclusion frameworks, for non-strong therapeutic items expected for inward breath and for parenteral or ophthalmic organization. In certain nourishment bundling material, Extractables and leachables contemplates are likewise required. The investigations ordinarily include uncovering an example of the material to a fitting dissolvable framework under pressure conditions to expand the pace of extraction. The dissolvable utilized for extraction ought to have indistinguishable affinity to remove substances from the dynamic substance/measurements frame as suitable. On account of therapeutic items, the favoured dissolvable would be the restorative item or fake treatment vehicle. The nature and measure of the separated substances ought to be recorded in the detail of the bundling material [24]. Extractables and leachables examines are done dependent on hazard based methodology for parenteral and ophthalmic medication items. USP compendial references $<661>,<1151>$ and $<601>$ ought to be entered on the Extractables and leachables study. Right now, populace, course of organization and potential for collaboration among definition and holder conclusion framework are considered.

The FDA has proposed PFS in the class of most noteworthy level of concern related with course of organization with high probability of cooperation between bundling segment dose structure. An exceptional case inside the Extractables and leachables study is the point at which the detailing is a lipid emulsion as it is increasingly inclined to associate with holder conclusion framework. These connections may influence basic parameters like globule size, strength, and so on. Other such models are high $\mathrm{pH}$ details. These plans may remove silicone oil from the holder that is commonly used to grease up the conclusion. High $\mathrm{pH}$ may likewise extricate follow metals from the plastic compartments. Diluents at times make issue with fogginess in the detailing planned for reconstitution. It is obligatory to check the similarity just as Extractables and leachables concentrate for diluents like Normal Saline, Ringer's answer, and so on. All out Parenteral Nutrition admixture parts must be tried for aluminium content as they are increasingly inclined to extricate from compartments. This can be a fundamental worry in glass holders. Overall, leachables are more basic than extractables [25]. To completely assess the danger of tungsten-prompted protein corruption in syringe barrels, the advancement group ought to describe the lingering tungsten level and investigate which tungsten species might be available. Inductivity-coupled plasma/mass spectroscopy innovation is very much created for examination of follow metals in watery arrangements and can be embraced promptly to gauge tungsten particle focus in extricate arrangements just as in medicate item. Nashed et al. have exhibited a stage way to deal with assessing potential leachables from a PFS framework [26].

A wide range of glass can possibly drain a scope of mixes into the item. The degrees of leachables found in the $\mathrm{pH}$ scope of are generally low, expanding fundamentally at $\mathrm{pH}$ more prominent than the major extractables found are sodium, barium, calcium and aluminium particles just as boron relying upon the particular glass arrangement. Conceivable follow leachables incorporate iron, magnesium and zinc particles. Draining of metal particles can be quickened by chelating operators, for example, EDTA bringing about potential glass pitting. Another pre-filled syringe-producing step that can prompt leachables is the glue procedure used to append the needle to staked-in needle pre-filled syringes. The UV light procedure, which is regularly used to fix the glue, might be inadequate prompting conceivably oxidizing dissolvable polluting influences. This hazard can, nonetheless, be significantly diminished by process upgrades at the syringe merchant. Various parts can likewise drain into the medication item from the elastic materials of the unclogger, including 2-mercaptobenzothiazole, aluminum or zinc cations or nitrosamines. Surfactant aided draining of a vulcanising specialist from uncoated plugs was proposed as a component adding to antagonistic clinical impacts and item disappointment of in a prefilled syringe group. The degrees of extractables from the elastic materials are ordinarily given by the producers. These levels can be limited by use of a reasonable covering, for example, poly (ethylene-co-tetrafluoroethylene) (ETFE) polytetrafluoroethylene (PTFE) [22].

\section{Challenges with PFS drug delivery system}

A few difficulties that are experienced with pre-filled syringes incorporate connections of the item with silicone as well as tungsten and development of the uncloggers during terminal sanitization or delivery. Glass syringes, uncloggers, needles and some polymeric syringes are covered with silicone oil. Siliconization alludes to the treatment of surfaces with polydimethylsiloxane. Polydimethylsiloxane is otherwise called silicone liquid. The liquid might be applied as silicone oil or as an oil in water emulsion. It is normally directed by splashing the liquid or emulsion onto a perfect, dry surface, however may likewise be applied by plunging or wiping [27]. The layer of silicone is air dried or prepared onto the outside of the material [27]. Needles are covered with silicone oil to 
diminish frictional drag while infiltrating tissue [28]. This assists with diminishing torment related with infusions. A covering of silicone is required on plugs to improve their machinability. The covering keeps the uncloggers from adhering to one another, from adhering to the hardware, improves their course through the container and arrangement into the syringes. Covering the uncloggers and syringes with silicone additionally diminishes erosion during use. Not as much weight is expected to start the development of the unclogger while conveying the portion to a patient and the unclogger will float all the more unreservedly through the barrel of the syringe. The objective is to apply simply enough silicone to accomplish the ideal oil that underpins machinability and simplicity of activity. No silicone or lacking utilization of silicone can bring about ending of the unclogger while conveying a portion. Tedious stopping of the unclogger in the barrel of the syringe is alluded to as prattling. The silicone oil in the syringe barrels and on the uncloggers makes two or three difficulties for items filled into the syringes. To start with, free silicone oil will be available in the medication arrangement as round beads and will be recognized as particulates by molecule tallying instruments. Organic items that are filled into the syringes may interface with the silicone and this may bring about the development of totals. Accumulation of proteins within the sight of silicone oil was considered. The gathering found that total was profoundly subject to the protein, the $\mathrm{pH}$ and the arrangement condition, yet could be limited by lessening the amount of silicone present 3 . Silicone oil beads were likewise announced as an issue in the facility while conveying an intravitreal infusion. The investigation exhibited that silicone beads were being conveyed into the eye and that they could be diminished if the infusion was conveyed utilizing a Luer conedesign versus a staked needle [29]. It was theorized that the staked syringe conveyed more silicone to the eye since it didn't have a dead space preceding arriving at the needle as on account of a Luer cone design[29]. This permitted the unclogger to skim a layer of silicone from the sides of the barrel and push it through the needle while conveying the whole portion present in the syringe [29].

Totals of proteins and monoclonal antibodies can likewise shape because of introduction to tungsten. Tungsten tests are utilized to shape the liquid way from the syringe barrel to the needle in syringes. The tests is added into the warmed glass and can desert tungsten build up. Conglomeration of a protein happened after presentation to tungsten in a syringe. An investigation revealed that the protein arrangement reached the tungsten saved in the liquid way of the syringe after uncloggers were embedded utilizing vacuum placement. Arrangement streamed into the liquid way during vacuum position of the stoppers. Conglomeration after presentation to tungsten, specifically, the tungsten species kept in the liquid way of glass syringes, was likewise detailed for epoetin alfa, a monoclonal neutralizer and other proteins [31]. Distinguishing tungsten as a reason for total can be troublesome. The amount of tungsten build up can fluctuate from barrel to barrel and parcel to lot [31]. The inconstancy will make it hard to recognize as an issue when testing arrangements in syringes at research facility scale. Syringes with staked needles present another test. The needles in pre-staked syringes are held set up with an UV-relieved cement. This raises the hazard for cooperation of the medication with the glue and additionally association with parts of the cement that might be extracted [31]. A test to the sterility of the substance of a prefilled syringe might be experienced during terminal cleansing and sending. The adjustments in pressure during sanitization and transportation can make the unclogger move into the non-sterile region of the syringe barrel and bargain the sterility of the substance when the unclogger comes back to the expected position. The development can be constrained or forestalled during terminal cleansing by diminishing the weight differentials during the activity. Maybe a superior technique for diminishing unclogger development is by controlling the tallness of the unclogger just as the air space between the unclogger and the clean fluid. Studies led by Kinney et al. exhibit that more unclogger development is normal as the air space between the unclogger and the clean fluid increments and as the stature of the unclogger decreases[33].

In light of the unclogger - barrel plan of PFS, syringe barrels must be covered with silicone to guarantee adequate and uniform coast power for the unclogger to handily convey the substance. Capacity of proteins in silicone-covered PFS can at times bring about silicone draining into the item. Additionally, protein-based medications (particularly those defined at low or high $\mathrm{pH}$ ) can filter or concentrate contaminants from PFS elastic plugs. To beat these difficulties, many syringe producers grew new silicone-covering methods and a specific silicone evaluation to diminish the probability of silicon filtering. Additionally, Pantheon's Pidgeon and Baxter's Chaubal recommended that a portion of the extractable worries with PFS may be overwhelmed by supplanting elastic plugs with less artificially responsive substances like Teflon. These can be executed as uniform covering of typical elastic plugs with Teflon, etc. Overall, glass has become the decision of material for PFS enterprises. It is important to search for choices as now and then delamination raises basic issues. Capacity in glass holders may ingrain the item with minuscule shards of glass in item arrangement of lower $\mathrm{pH}$ after some time. These have driven them to search for different alternatives, for example, COC, COP or Crystal Zenith. They are low with respect to being Extractables and leachables and stable over wide pH extend. One significant test to across the board acknowledgment of PFSs as the favoured conveyance framework is the human brain science of not evolving without any problem. Vials and ampoules have been standard for a considerable length of time, implying that a changeover to an elective strategy will take both powerful correspondence and conduct change to end clients. Additionally, solidness is a valid statement to cite here. Time span of usability $<2$ years implies a weight in 
expenses of item because an item takes up to a half year to experience its circulation chain. Accordingly, any item having lower strength is useless to create in PFS. Overall, there is a recognition by and large that regulating drugs in PFS group is expensive. On the off chance that one sees and looks at the regulatory, work force and subordinate expenses related with organization of biologics and immunizations in vials and pre-fills framework, the later offers genuine cost advantage over the other notwithstanding numerous different advantages.

\section{REFERENCES}

[1] Harrison B, Rios M. Developments in pre-filled syringes. Pharm Techol 2007; 31(3):50-60

[2] Overcashier D, Chan E, Hsu C. Technical considerations in the development of pre-filled syringes for protein products. Am Pharm Rev 2006; 9: p.77-82

[3] The lucrative challenges of pre-filled syringe technology. 2013. Available from: http://blog. pharmatechnologyindex.com/2013/01/thelucrative-challenges-of-pre-filledsyringe technology

[4] FDA Staff. Glass syringes for delivering drug and biological products: technical recommendations to supplement International Organization for Standardization (ISO) Standard 11040-4. 2013. Available from: www.fda.gov/

[5] Vaczek D. Promoting dosing accuracy with prefilled syringes. Pharm Med Pack News 2007; 15: p. 42-50

[6] Kiang P. Future materials for pre-filled syringe components. Am Pharm Rev 2011; 14:54-8

[7] Sacha GA, Saffell-Clemmer W, Abram K, et al. Practical fundamentals of glass, rubber, and plastic sterile packaging systems. Pharm Dev Technol 2010; 15:6-34

[8] Wakankar AA, Wang YJ, Canova-Davis E, et al. On developing a process for conducting extractable-leachable assessment of components used for storage of biopharmaceuticals. J Pharm Sci 2010; 99:2209-18

[9] Jenke D. Evaluation of the chemical compatibility of plastic contact materials and pharmaceutical products; safety considerations related to extractables and leachables. J Pharm Sci 2007; 96:2566-81

[10] Adler M. Challenges in the development of pre-filled syringes for biologics from a formulation scientist's point of view. Am Pharm Rev 2012;15:1-8

[11] Romacker MTS, Forster R. The rise of prefilled syringes from niche product to primary container of choice: a short history. ON drug Delivery 2008; 4:4-5

[12] Badkar A, Wolf A, Bohack L, et al. Development of biotechnology products in pre-filled syringes: technical considerations and approaches. AAPS Pharm SciTech 2011; 12:564-72

[13] Felsovalyi F, Janvier S, and Jouffray S, et al. Silicone-oil-based sub visible particles: their detection, interactions, and regulation in prefilled container closure systems for biopharmaceuticals. J Pharm Sci 2012; 101:4569-83

[14] Baldwin RN. Contamination of insulin by silicone oil: a potential hazard of plastic insulin syringes. Diabet Med 1988; 5:789-90

[15] Thirumangalathu R, Krishnan S, Ricci MS, et al. Silicone oil- and agitation-induced aggregation of a monoclonal antibody in aqueous solution. J Pharm Sci 2009; 98:3167-81

[16] United States Pharmacopeia general chapter 788. Particulate Matter in Injections

[17] Ludwig DB, Carpenter JF, Hamel J-B, et al. Protein adsorption and excipient effects on kinetic stability of silicone oil emulsions. J Pharm Sci 2010; 99:1721-33

[18] Sluzky V, Tamada JA, Klibanov AM, et al. Kinetics of insulin aggregation in aqueous solutions upon agitation in the presence of hydrophobic surfaces. Proc Nat Acad Sci USA 1991; 88:93 77-81

[19] Jiang Y, Nashed-Samuel Y, Li C, et al. Tungsten-induced protein aggregation: solution behaviour. J Pharm Sci 2009; 98:4695-710

[20] Waxman L, Steeley T, Vilivalam V. Protein sensitivity to tungsten. Pharma Tech 2011; 28-32

[21] Seidl A, Hainzl O, Richter M, et al. Tungsten-induced denaturation and aggregation of epoetin alfa during primary packaging as a cause of immunogenicity. Pharm Res 2012; 29:14 54-67

[22] Sacha GA, Saffell-Clemmer W, Abram K, et al. Practical fundamentals of glass, rubber, and plastic sterile packaging systems. Pharm Dev Technol 2010; 15:6-34

[23] Manek SP. Extractables and leachable regulatory perspective. IPA Pharma; Mumbai, India; 2011

[24] Guideline on plastic immediate packaging materials. $2009 . \quad$ Available from: www.ema.europa.eu/docs/en_GB/document_library/Scientific_guideline/2009/09/WC500003448.pdf: www.ema.europa.eu/

[25] Lewis DB. Current FDA perspective on leachable impurities in parenteral and ophthalmic drug products. AAPS Workshop on Pharmaceutical Stability-Scientific and Regulatory Considerations for Global Drug Development and Commercialization. Office of New Drug Quality Assessment CDER/FDA; Washington DC, USA: 2011

[26] Nashed SY, Silva EM, Swift R, Bordas NJ. Strategies for baseline extractables evaluation of a pre-filled syringe delivery system for water based biopharmaceutical formulations. Parenteral Drug Association. Extractables and Leachables Forum: The Extractables Puzzle: An Integrated Team Approach, Bethesda, MD; 2005.

[27] Colas A, Siang J, Ulman K. Silicones in pharmaceutical applications. Part 5: siliconization of parenteral packaging components. Midland (MI): Dow Corning Corporation; 2006.

[28] PDA. Siliconization of parenteral drug packaging components (Technical Report No. 12). J Parenteral Sci Technol 1988; 42 : S1-S13.

[29] Scott IU, Oden NL, Vanveldhuisen PC, and et al. SCORE study peport 7: incidence of intravitreal silicone oil droplets associated with stakedon vs. Luer cone syringe design. Am J Ophthamol 2009 148: 725-732.

[30] Jiang Y, Nashe-Samuel Y, Li C, et al. Tungsten-induced protein aggregation: solution behavior. J Pharm Sci 2009; 98:4695-4710.

[31] Seidl A, Hainzl O, Richter M, et al. Tungsten-induced denaturation and aggregation of epoetin alfa during primary packaging as a cause of immunogenicity. Pharm Res 2012; 29:1454-1467.

[32] Swift R. Prefilled syringes for biopharmaceuticals. Formulation and process development strategies for manufacturing biopharmaceuticals. Hoboken (NJ): John Wiley \& Sons, Inc.; 2010:897-916.

[33] Kinney S, Wagner A, Phillips C. A rational approach to determining the maximum allowable gas bubble inside a prefilled syringe to minimize stopper movement \& protect product sterility. Drug Delivery Technol 2009; 9:42-47. 